\title{
Irradiating of children: whose responsibility?
}

No one in the practice of medicine today can deny the incredible advances and impact that computed tomography has had on how we display anatomy and visualise pathology. We entered the 21 st century with over 60 million CT studies a year in the USA - and that number climbing. Spiral or helical technology meant greater coverage in less time, and multiple scans related to contrast administration became standard. Increasing availability of CT scanners allowed increasing utilisation. The annual increase of approximately $10 \%$ in CT studies has occurred with only a $1 \%$ population increase. Of the 377 million diagnostic and interventional studies performed in the USA in 2006, 67 million were CT scans - yet these CT scans contributed $49 \%$ of all medical radiation dose.

CT studies alone deliver an annual $1.47 \mathrm{mSv}$ per capita to the USA population (the annual background of $3 \mathrm{mSv}$ is without this additional medical radiation). These figures are from published literature, and I am not aware of any similar published figures from South Africa, but I strongly suspect that very similar trends in CT usage have occurred anywhere that CT scanners are available and can be afforded.

For all the advantages that CT has generated, there has been a significant hidden cost - radiation. A CT study has a high individual radiation burden but, collectively, the increased exposure to the population at large may well be a larger future concern.

$\begin{array}{ll}\text { Some basic radiation values to guide us are: } \\ \text { Frontal CXR } & 0.01 \mathrm{mSv} \\ \text { Lung CT } & 3 \mathrm{mSv} \\ \text { Abdominal CT } & 10 \mathrm{mSv} \\ \text { Annual background radiation } & 3 \mathrm{mSv}\end{array}$

Radiation effects can be deterministic, requiring a threshold dose to manifest. Examples are cataract formation and skin injury, both requiring large exposure, above $2000 \mathrm{mSv}$. Knowing these thresholds easily allows setting limits and removing the risk. Of more concern are the stochastic or random biological effects of radiation on DNA. They do not have known thresholds, and the BEIR VII reports indicate a linear related response from very low exposure to radiation. Ionisation can occur directly with electrons of DNA molecules but, more commonly, radiation interaction with water molecules creates hydroxyl radicals, which then interact with DNA. Single-chain damage is usually quickly repaired but double-chain damage may be difficult to

After completing his diagnostic radiology training at Tygerberg Hospital (University of Stellenbosch) in 1991, Dr Jamieson started his paediatric radiology career under the guidance of Professor Bryan Cremin at Red Cross War Memorial Children's Hospital in Cape Town. His paediatric fellowship training was at The Hospital for Sick Children in Toronto, Canada. Since 1996, he has been a paediatric radiologist at BC Children's Hospital in Vancouver and an associate professor in the Department of Radiology at the University of British Columbia. Mountaineering, skiing, river running and sea kayaking remain avid interests outside of paediatric radiology. repair. The consequence may be radiation-induced carcinogenesis in the exposed individual but, also, this 'radiation-induced genomic instability' can be transmitted to future generations! Much has been learnt of these stochastic radiation effects from the intensive epidemiological study of nuclear bomb survivors from Nagasaki and Hiroshima in 1945. Longterm and robust data have shown increased cancers in survivors exposed to radiation doses equivalent to a modern CT study. The cancers include leukaemia, breast, digestive organs, colon, thyroid and lung.

Of particular concern is the age-related effect of radiation. The younger the subject, the higher the radiation-induced effects. Children are deemed more radiosensitive as they have a larger proportion of actively dividing cells, and they have a longer latent period in which a radiation-induced cancer has the opportunity to manifest. Young females are also noted to be more radiosensitive than males.

At my paediatric institution, we estimate the lifetime risk of a future cancer as a result of a paediatric CT to be about 1:1000. This is an approximation. It is, however, clear that it has become our duty as physicians and radiologists to utilise CT scanning in children wisely and appropriately.

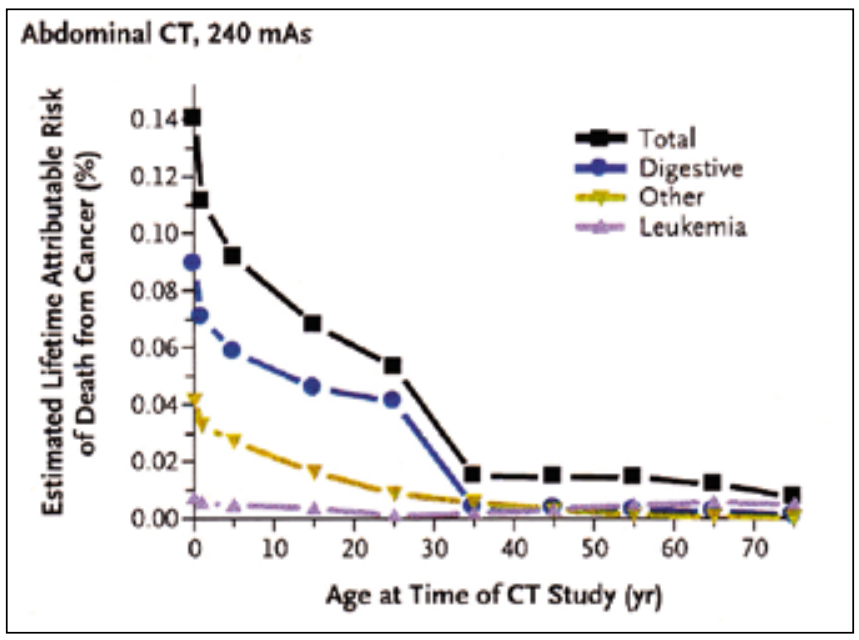

N Engl J Med 2007;357:2277-2284.

\section{Action}

How many paediatric CT scans have you reported in the last year that had uncertain indications or would not clearly influence patient management and diagnosis? Reports in the literature from 2002 and 2005 indicate that $30-40 \%$ of paediatric CT scans are not justified by medical need! Given what we know today about the effects of radiation in children, this excess of CT imaging must be curtailed. Improved screening of $\mathrm{CT}$ requests is, I believe, fairly and squarely the responsibility of the radiologist performing or interpreting the resultant examination.

If $\mathrm{CT}$ is the best and most appropriate imaging required, then paediatric-appropriate protocols and techniques are mandatory. Sizeand weight-related reduction in $\mathrm{kVp}$ and $\mathrm{mAs}$, appropriate collimation and table speed can substantially reduce the radiation dose. Reducing 


\section{EDITORIAL}

anatomical coverage to the relevant area and excluding multiphase acquisitions, unless a clear advantage is to be gained, should become normal practice.

Claiming ignorance and lack of knowledge for not instituting change at any facility imaging children is no longer acceptable. There is an excellent source of information with paediatric-specific guidelines, and protocols are freely available on the Internet. I strongly encourage every radiologist imaging children to visit and explore the Society for Pediatric Radiology's web-based initiative: Image Gently - The Alliance for Radiation Safely in Pediatric Imaging (Google 'Image Gently' or access the website at http://www.pedrad.org/associations/5364/ig/). It is also pertinent to note that this excellent web site not only provides the radiologist with information on paediatric-specific protocols and how to implement them in one's practice, but it is also readily available to one's patients and their parents to learn about the risks and benefits of diagnostic imaging.

We should always ask if there are alternatives to radiation in the diagnostic imaging of children.

Ultrasound. In neonates and small children, the spatial resolution of high-resolution linear probes cannot be matched by CT or MR! Ultrasound is a very good tool for the evaluation of appendicitis in paediatrics. CT may perform slightly better (certainly an advantage in the obese patient), but does this mean that anyone with right iliac fossa (RIF) pain in the ER needs a CT abdomen before they see a surgeon? Surely a good clinical evaluation with US as a primary imaging tool is an appropriate balance between diagnosis and radiation burden. No one would question the role for CT in equivocal or diagnostic dilemma situations.

Magnetic resonance. Its increased use in paediatrics is limited largely by availability and cost. Hardware and technical improvement have made 'breath hold imaging' routine. Where available, MR has replaced neonatal brain imaging. MR imaging of musculoskeletal pathology is established, and MR has gained a large component of abdominal imaging including solid organ tumour evaluation, the biliary tree and bowel diseases such as IBD.

\section{Conclusion}

$\mathrm{CT}$ is a great tool and has revolutionised diagnostic imaging, but it should be used wisely, especially in paediatric patients where the radiation burden has the most impact and consequence. There are alternatives to consider, in particular US and MR.

Do not allow your patient to become better informed about radiation than you might be; visit the Image Gently web site for information.

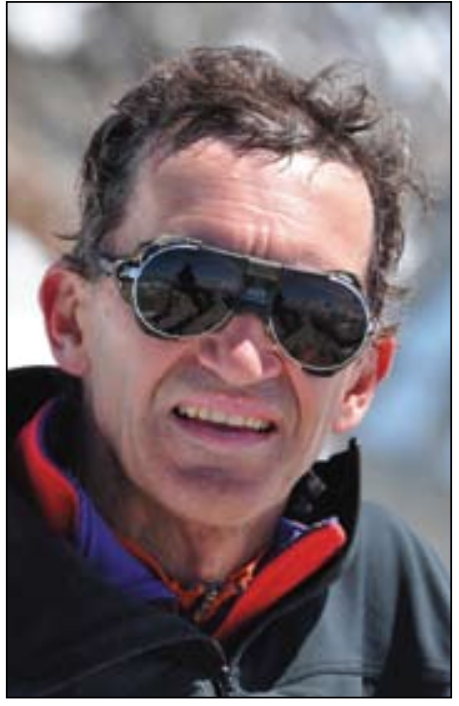

It is our responsibility as radiologists to monitor, utilise and advise on the use of diagnostic radiation, especially in children. Education of referring clinicians regarding appropriate use of diagnostic imaging is a challenge that we should meet with dialogue, collaboration, information, and compliance with the principle that all doctors should identify with: Primum non nocere (Above all, do no harm.).

\section{Douglas H Jamieson \\ Paediatric Radiologist \\ BC Children's Hospital \\ Vancouver, Canada; and \\ Associate Professor \\ Department of Radiology \\ Faculty of Medicine \\ University of British Columbia \\ djamieson@cw.bc.ca}

Recommended reading

Brenner DJ, Hall EJ. Computed tomography - an increasing source of radiation exposure. N Eng J Med 2007;357:2277-2284.

Donnelly LF. Reducing radiation dose associated with pediatric CT by decreasing unnecessary examinations. AJR 2005;184:655-657.

Donnelly LF, Emery KH, Brody AS. Minimizing radiation dose for pediatric body applications of singledetector helical CT: strategies at a large children's hospital. AJR 2001;176:303-306.

Hall EJ. Lessons we have learned from our children: cancer risks from diagnostic radiology. The 2002 Neuhauser Lecture. Pediatr Radiol 2002;32:700-706.

Mettler FA, Bhargavan M, Faulkner K, et al. Radiological and nuclear medicine studies in the United States and worldwide: Frequency, radiation dose, and comparison with other radiation sources 1950 - 2007. Radiol 2009;253:520-531.

National Research Council of The National Academies. BEIR VII: Health Risks from Exposure to Low Levels of Ionizing Radiation. National Academies Press, Washington DC: 2005.

Pierce DA, Preston DL. Radiation-related cancer risks at low doses among atomic bomb survivors. Radiat Res 2000;154:178-186.

Slovis T. CT and computed radiography: The pictures are great, but is the radiation dose greater than required? AJR 2002;179:39-41. 